\title{
Anticancer Evaluation of Secondary Metabolites in Hep- 2 Cell Lines from Exiguobacterium Profundum BC2-11
}

\section{Mani VM*1\&2 and Preethi $\mathbf{K}^{1}$}

${ }^{1}$ Department of Microbial Biotechnology, Bharathiar University, India

${ }^{2}$ Department of Biotechnology, Hindusthan College of Arts \& Science, India

*Corresponding author: Manon Mani Vellingiri, College Assistant Professor,

\section{Research Article}

Volume 1 Issue 1

Received Date: August 11, 2016

Published Date: September 10, 2016

DOI: $10.23880 /$ oajmb-16000106

Department of Biotechnology, Hindusthan College of Arts \& Science, Coimbatore, TN, India, Tel: 09715250576 ; E-mail: manonmanisathee12@gmail.com

\section{Abstract}

The stressed environment contains full of oxidative stress which constitutes the plants and microbes. These sustains in this environment by changing its biological activity by producing some of the metabolites as intermediate products to survive within the microbial population. As these produce different metabolites with different biological activities so this current investigation has focused on the characterization and biological evaluation of the potential metabolite from $E$. profundum BC2-11 which was isolated from the stressed environment. The crude pigment extract was subjected to HPTLC profile and it exhibited six peaks, the $5^{\text {th }}$ peak was observed to be highest area of $5973.3(47.39 \%)$ which was the bioactive metabolite. The crude colored metabolite was assessed for antimicrobial assessment against clinical pathogens and it revealed highest activity against maximum pathogens. The radical scavenging activity for the colored metabolite was determined through DPPH, metal chelation, reductive power and total antioxidant capacity. The colored metabolite disclosed $50 \%$ of inhibitory activity at lower concentrations. The in vitro cytotoxicity evaluation was analyzed in Hep-2 cell lines and the dilution of 1:4 indicated about $54 \%$ cell viability and this was confirmed through MTT assay. Further the colored metabolite will be taken for bioprospecting studies and pharmaceutical applications.

Keywords: Anticancer; Antioxidant; Cytotoxicity; HPTLC; MTT

\section{Introduction}

Pollution is the effect of undesirable changes in our surroundings that have harmful effects on plants, animals and human beings. This occurs when only short-term economic gains are made at the cost of the long-term ecological benefits for humanity. No natural phenomenon has led to greater ecological changes than have been made by mankind. The most of soil microbes are functionally redundant and replaceable with other species without any change in general soil function. According to recent estimation, the reduction of taxonomic diversity in metal polluted soils can reach $99.9 \%$, with most species belonging to rare taxa, but the functional importance of these rare taxa for soil nutrient cycling and ecosystem resilience is unknown. Changes in the relative diversity and functional characteristics of microbial communities might have unpredictable consequences for nutrient cycling and thus productivity. A research has found the soil bacterial functional diversity to be a good indicator of 
effects in different metal pollution, ecosystem type or ecosystem management. The changes in the abiotic factors (temperature, moisture) may stronger affect less diverse soil microbial communities. Resistance of the microbial communities in long term polluted soils (e.g. heavy metals) to an additional stressors and/or disturbances (temperature, soil moisture, toxic compounds). A large numer of bacteria, fungi and algae produce pigments. These pigments are biological pigments and can be used for various industrial purposes. Bacteria are found to contain more anticancer property which induces the apoptosis in cancer cells and induces the cell death. With this view, the research has been focused to characterize the propsective secondary metabolites extract and evaluate its cytotoxicity properties in Hep-2 cell lines. This would reveal the anticancer property for the secondary metabolites extract from E. Profundum BC2-11 (Genebank accession No: KP966110) [1].

\section{Materials and Methods}

\section{Antioxidant Activity}

DPPH radical scavenging assay: The effect of colored metabolite extract on DPPH radical was determined using the method of Mani et al (2015). Different concentrations of the colored metabolite extract ( 50 to $100 \mu \mathrm{g} / \mathrm{ml}$ ) were prepared and subjected to antioxidant tests. To $1 \mathrm{ml}$ of each concentration of the extract, $5 \mathrm{ml}$ of $0.1 \mathrm{mM}$ methanol solution of DPPH was added, vortexed and followed by incubation at $27^{\circ} \mathrm{C}$ for $20 \mathrm{~min}$. The control was prepared without any extract and absorbance of the sample was measured at $517 \mathrm{~nm}$ using UV/VIS Spectrophotometer using methanol to set 0 . The ability to scavenge DPPH radical was calculated by the following equation: DPPH scavenging effect $(\%)=\left[\left(A_{0}-A_{1}\right) / A_{0}\right] \times 100$; Where $A_{0}$ was the absorbance of the control reaction and $A_{1}$ was the absorbance in the presence of the standard sample or fraction.

As the secondary metabolites extract contain rich in antioxidants, the extract was taken for cytotoxicity evaluation through MTT analysis.

\section{In vitro Cytotoxicity Analysis}

The Hep- 2 cell lines was were purchased from NCCS Pune were maintained in Dulbecco's modified eagles media (HIMEDIA) supplemented with 10\% FBS (Invitrogen) and grown to confluency at $37^{\circ} \mathrm{c}$ in $5 \% \mathrm{CO}_{2}$ in a humidified atmosphere in a $\mathrm{CO}_{2}$ incubator(NBS, Eppendorf, Germany). The cells were trypsinized (500 $\mu \mathrm{l}$ of $0.025 \%$ Trypsin in PBS/ $0.5 \mathrm{mM}$ EDTA solution
(Himedia)) for 2 minutes and passaged to T flasks in complete aseptic conditions. For the assays and the continuous cell propagation adherent monolayers in exponential growth phase (about $80 \%$ confluence) were harvested with trypsin/ EDTA $(0.05 \% / 0.02 \%)$ in phosphate buffered saline (PBS).

\section{MTT Assay}

MTT is a colorimetric assay that measures the reduction of yellow 3-(4, 5dimethythiazol-2-yl)-2, 5diphenyl tetrazolium bromide (MTT) by mitochondrial succinate dehydrogenase. The MTT enters the cells and passes into the mitochondria where it is reduced to an insoluble, coloured (dark purple) formazan product. The cells are then solubilised with an organic solvent Dimethyl sulfoxide (Himedia) and the released, solubilised formazan product was measured at $540 \mathrm{~nm}$. Since reduction of MTT can only occur in metabolically active cells the level of activity is a measure of the viability of the cells. $6.25,12.5,25,50,100 \mu \mathrm{g} / \mathrm{ml}$ of sample was added from $1 \mathrm{mg} / \mathrm{ml}$ stock to grown cells and incubated for 24 hours. The \% difference in viability was determined by standard MTT assay after 24 hours of incubation.

The cells were washed with $1 \mathrm{x}$ PBS and then added $30 \mu \mathrm{l}$ of MTT solution to the culture (MTT $-5 \mathrm{mg} / \mathrm{ml}$ dissolved in PBS). It was then incubated at $37^{\circ} \mathrm{C}$ for 3 hours. MTT was removed by washing with $1 \mathrm{x}$ PBS and $200 \mu \mathrm{l}$ of DMSO was added to the culture. Incubation was done at room temperature for 30 minutes until the cell got lysed and colour was obtained. The solution was transferred to centrifuge tubes and centrifuged at top speed for 2minutes to precipitate cell debris. Optical density was read at $540 \mathrm{~nm}$ using DMSO as blank in a micro plate reader (ELISASCAN, ERBA). $\%$ viability $=(O D$ of Test/ OD of Control) X 100.

\section{Results}

\section{DPPH Antioxidant Assay}

DPPH free radical determines the free radical scavenging capacity or antioxidant potential (AOD) of the pigment sample, which shows its effectiveness by prevention, interception and repair mechanisms against injury in a biological system [1]. In this investigation, the $\mathrm{IC}_{50}$ value of colored metabolite extract was found to be $870 \mu \mathrm{g} / \mathrm{mL}$ concentration. It means that the colored metabolite had good scavenging activity at least concentration which is equivalent to standard ascorbic acid (Figure 1). 


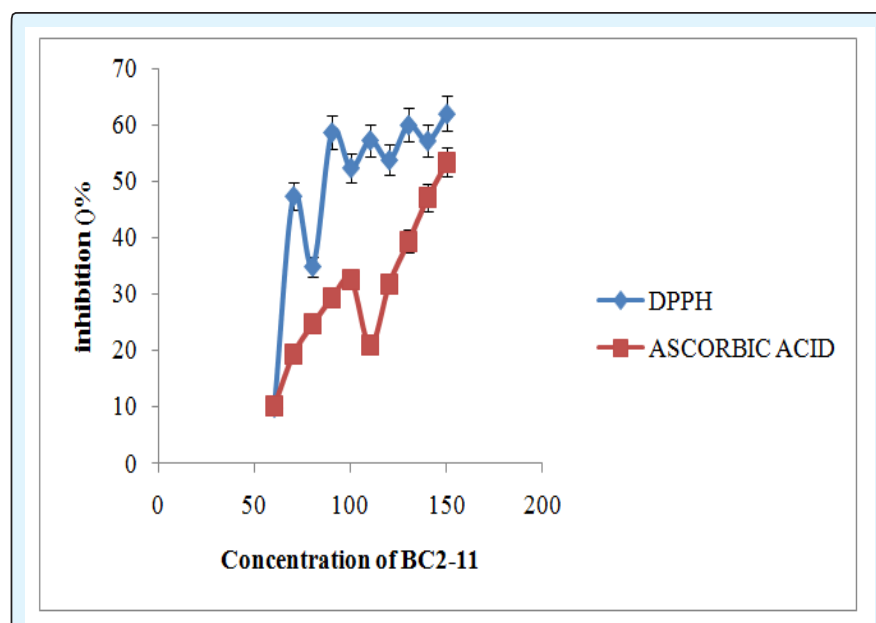

Figure 1: DPPH analysis of secondary metabolites extract from E. profundum BC2-11.

\section{Cytotoxicity Analysis}

\section{MTT Analysis}

The MTT analysis will reveal the cell death and cell viability at different concentration of the extract which was done in duplicates. In this investigation the sample was diluted at different concentration such as $0.2,0.4,0.6$, $0.8,1.0,1.2$ and $1.4 \mu \mathrm{g} / \mathrm{mL}$ (Table 1 and Figure 2). The secondary metabolites extract was found to inhibit maximum cells at a moderate concentration. At the concentration of $1: 4$ the cell viability was found to be $54.38 \%$ which constitutes the compound of about $0.6 \mu \mathrm{g}$ (Figure 3). This exhibited the maximum inhibition of the extract at minimum concentration.

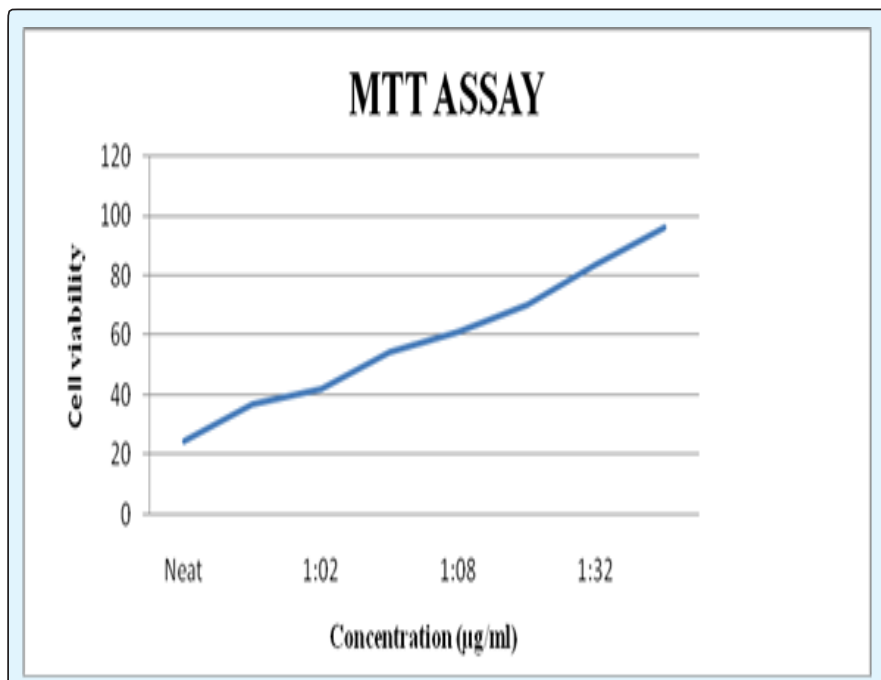

Figure 2: MTT analysis of secondary metabolites extract from E. profundum BC2-11.
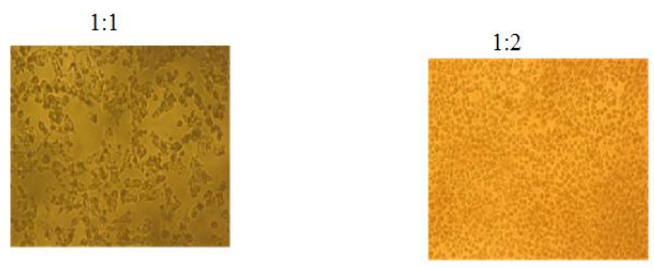

$1: 4$

$1: 8$
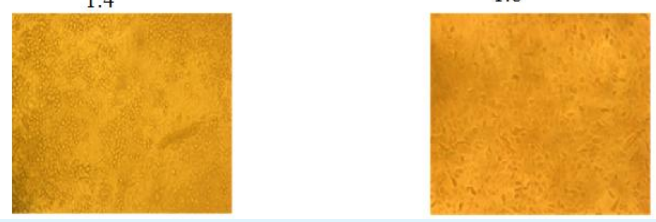

Figure 3: MTT analysis of secondary metabolites extract from E. profundum BC2-11.

\begin{tabular}{|c|c|c|c|}
\hline S.no & Dilutions & Absorbance & Cell viability \\
\hline 1 & Neat & 0.14 & 24.56 \\
\hline 2 & $1: 1$ & 0.21 & 36.84 \\
\hline 3 & $1: 2$ & 0.24 & 42.10 \\
\hline 4 & $1: 4$ & 0.31 & 54.38 \\
\hline 5 & $1: 8$ & 0.35 & 61.40 \\
\hline 6 & $1: 16$ & 0.40 & 70.17 \\
\hline 7 & $1: 32$ & 0.48 & 84.21 \\
\hline 8 & $1: 64$ & 0.55 & 96.49 \\
\hline 9 & Cell control & 0.57 & 100 \\
\hline
\end{tabular}

Table 1: MTT analysis of secondary metabolites extract from E. profundum BC2-11.

\section{Discussion}

The bacterial strains were found to inhabitant on soil environment. The iron and steel industry causes significant effects on environmental media such as air emissions, soil pollution by sedimentation and water pollution of settling up of organic matter, oil, grease, suspended solids, benzene, phenols, acids and ammonia. These interact with the soil community and the community includes plants, microbes and water source. These conditions drastically affect the microbe which leads to produce different molecules and metabolites for their survival between the microbial communities. Because of these serious problems the micro organisms were able to develop different pathways for synthesizing different metabolites [1]. This strain E. profundum BC2-11 was reported to produce the carotenoids [2]. The colored metabolite was extracted using methanol when compared 
to other solvents. The stressed environmental soil had majority of micro organisms which were prevalent in producing different metabolites for their survival between microbial communities. The isolated Exiguobacterium profundum BC2-11 produced orangish metabolite which possessed the antimicrobial as well as possesses the antioxidant property. This secondary metabolites extract was taken for anticancer evaluation through MTT analysis. The different dilution of the extract was assessed and cell viability ranged as $54 \%$ at $1: 4$ diluted concentrations. The cells will enter the $\mathrm{G}_{0} / \mathrm{G}_{1}$ phase where the proliferation starts and the cell which are not in complete morphology will be neglected in this stage. The complete cells will enter the $S$ phase where the genetic materials will be synthesized and the cell will pass on to $G_{2}$ and $M$ phase where mitosis taken place with the division. The cells will get multiplied at this stage. Most of the secondary metabolites attack the $G_{1}$ phase so that cells will not enter $S$ phase and these will be dead at this phase itself. Whereas most of the anticancer metabolites induce the apoptosis in S phase which would eventually stop the growth and further processes in a cell. So the cell viability will be less when compared to other non cancerous drugs. In this investigation, the secondary metabolites extract profoundly induce apoptosis at G phase and further confirmation have to be determined through FACS analysis. So we suggest that this orangish metabolite can be taken as anticancer drug in medical fields.

\section{References}

1. Manon Mani V, Keerthana G and Preethi K (2015) Evaluation of antioxidant potential of bioactive colored metabolite isolated from Exiguobacterium profundum BC2-11 and it's bioactivities. Int J Recent Sci Res 6(4): 3612- 3617.

2. Fatima S, Hoda Y, Hanafy H (2013) Pigment production by Exiguobacterium aurantiacum $\mathrm{FH}$, a novel Lebanese strain. Int J Curr Microbiol App Sci 2(12): 176-191.

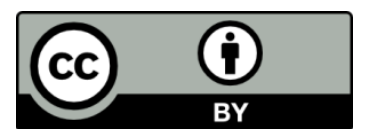

